International Journal of Pure and Applied Mathematics

Volume 97 No. 2 2014, 195-200

ISSN: 1311-8080 (printed version); ISSN: 1314-3395 (on-line version)

url: http://www.ijpam.eu

doi: http://dx.doi.org/10.12732/ijpam.v97i2.7

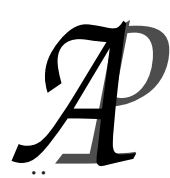

ijpam.eu

\title{
SOME APPLICATION OF PRIME IDEALS OF AG-RING
}

\author{
T. Gaketem \\ Department of Mathematics \\ School of Science \\ Phayao University \\ Phayao, 56000, THAILAND
}

Abstract: In this paper we define c-prime, 3-prime and weakly prime ideal of AG-ring which we will study relation of c-prime, 3-prime, weakly prime ideal and prime ideal.

Key Words: c-prime, 3-prime, weakly prime ideal

\section{Introduction}

M.A. Kazim and MD. Naseeruddin [2, Proposition 2.1] asserted that, in every LA-semigroups $G$ a medial law hold

$$
(a \cdot b) \cdot(c \cdot d)=(a \cdot c) \cdot(b \cdot d), \quad \forall a, b, c, d \in G .
$$

Q. Mushtaq and M. Khan [4, p.322] asserted that, in every LA-semigroups $G$ with left identity

$$
(a \cdot b) \cdot(c \cdot d)=(d \cdot c) \cdot(b \cdot a), \quad \forall a, b, c, d \in G .
$$

Further M. Khan, Faisal, and V. Amjid [3], asserted that, if a LA-semigroup $G$ with left identity the following law holds

$$
a \cdot(b \cdot c)=b \cdot(a \cdot c), \quad \forall a, b, c \in G .
$$

Received: June 19, 2014

(c) 2014 Academic Publications, Ltd. url: www.acadpubl.eu 
M. Sarwar (Kamran) [5, p.112] defined LA-group as the following; a groupoid $G$ is called a left almost group, abbreviated as LA-group, if $(i)$ there exists $e \in G$ such that $e a=a$ for all $a \in G$, (ii) for every $a \in G$ there exists $a^{\prime} \in G$ such that, $a^{\prime} a=e,($ iii $)(a b) c=(c b) a$ for every $a, b, c \in G$.

S.M. Yusuf in [6, p.211] introduces the concept of a left almost ring (LAring). That is, a non-empty set $R$ with two binary operations "+" and "." is called a left almost ring, if $\langle R,+\rangle$ is an LA-group, $\langle R, \cdot\rangle$ is an LA-semigroup and distributive laws of "." over "+" holds. T. Shah and I. Rehman [6, p.211] asserted that a commutative ring $\langle R,+, \cdot\rangle$, we can always obtain an LA-ring $\langle R, \oplus, \cdot\rangle$ by defining, for $a, b, c \in R, a \oplus b=b-a$ and $a \cdot b$ is same as in the ring. We can not assume the addition to be commutative in an LA-ring. An LA-ring $\langle R,+, \cdot\rangle$ is said to be LA-integral domain if $a \cdot b=0, a, b \in R$, then $a=0$ or $b=0$. Let $\langle R,+, \cdot\rangle$ be an LA-ring and $S$ be a non-empty subset of $R$ and $S$ is itself and LA-ring under the binary operation induced by $R$, the $S$ is called an LA-subring of $R$, then $S$ is called an LA-subring of $\langle R,+, \cdot\rangle$. If $\mathrm{S}$ is an LA-subring of an LA-ring $\langle R,+, \cdot\rangle$, then $S$ is called a left ideal of $R$ if $R S \subseteq S$. Right and two-sided ideals are defined in the usual manner. An ideal $I$ of $R$ is called prime if $A B \in I$ implies $A \in I$ or $B \in I$.

In this note we prefer to called left almost rings (LA-rings) as Abel-Grassmann rings (abbreviated as an "AG-rings").

In [1] An ideal $I$ of $N$ is called c-prime if $a, b \in N$ and $a b \in I$ implies $a \in I$ or $b \in I$. $R$ is called c-prime nearring if $\{0\}$ is a c-prime ideal of $R$.

An ideal $I$ of $R$ is called 3-prime if $a, b \in N$ and $a n b \in I$ for all $n \in N$ implies $a \in I$ or $b \in I$.

The notions of c-ideal, 3-prime ideal and prime ideal coincide in rings.

In [7] A proper ideal $I$ of an ring $R$ to be weakly prime if $0 \neq A B \subseteq I$ implies either $A \subseteq I$ or $B \subseteq I$ for any ideals $A, B$ of $R$.

The following implications are well known in rings:

(1) c-prime ideal $\Rightarrow 3$-prime ideal $\Rightarrow$ prime ideal;

(2) prime ideal $\Rightarrow$ weakly prime ideal.

\section{Main Results}

In this paper, we define c-prime and 3-prime of AG-ring.

Definition 2.1. An ideal $I$ of an AG-ring $R$ is called c-prime if $a, b \in R$ and $a b \in I$ implies $a \in I$ or $b \in I$. 
Definition 2.2. An ideal $I$ of an AG-ring $R$ is called 3-prime if $a, b \in R$ and $a r b \in I$ for all $r \in R$ implies $a \in I$ or $b \in I$.

The following lemmas and theorem we will study relation of c-prime, 3prime and prime ideals.

Lemma 2.3. Every c-prime ideal is a 3-prime ideal

Proof. Suppose that $I$ is a c-prime ideal of AG-ring $R$, let $a, b \in R$ and arb $\in I$ for all $r \in R$. Since $I$ is a c-prime ideal we have $a \in I$ or $b \in I$. Then $I$ is a 3 -prime ideal of $R$.

Lemma 2.4. Every 3-prime ideal is a prime ideal

Proof. Suppose that $I$ is a 3-prime ideal of AG-ring $R$, let $a, b \in I$ and $a b \in I$. Since $I$ is a 3 -prime ideal we have $a \in I$ or $b \in I$. Then $I$ is a prime ideal of $R$.

Lemma 2.5. Every c-prime ideal is a prime ideal

Proof. Suppose that $I$ is a c-prime ideal of AG-ring $R$, let $a, b \in I$ and $a b \in I$. Since $I$ is a c-prime ideal we have $a \in I$ or $b \in I$. Then $I$ is a prime ideal of $R$.

In [6, p.221]. studied if $I$ is a prime ideal in AG-ring $R$ if and only if $R / I$ is an AG-integral domain. The following theorems are application by lemmas 2.4 and 2.5

Theorem 2.6. Let $R$ be an AG-ring. Then $I$ is a 3-prime ideal in $R$ if and only if $R / I$ is an AG-integral domain.

Proof. $(\Rightarrow)$ Let $I$ is a 3-prime ideal in $R$. By Lemma 2.4 then $I$ is a prime ideal. Thus $R / I$ is an AG-integral domain.

$(\Leftarrow)$ Assume that $R / I$ is an AG-integral domain with $a r b \in I$ for all $r \in R$. Then $I+a r b=I$ so $(I+a) r(I+b)=I$. Since $R / I$ is an AG-integral domain we have $I+a=I$ or $I+b=I$. Then $a \in I$ or $b \in I$. Thus $P$ is a 3 -prime ideal of $R$.

Theorem 2.7. Let $R$ be an AG-ring. Then $I$ is a c-prime ideal in $R$ if and only if $R / I$ is an AG-integral domain. 
Proof. $(\Rightarrow)$ Let $I$ is a c-prime ideal in $R$. By Lemma 2.5 then $I$ is a prime ideal. Thus $R / I$ is an AG-integral domain.

$(\Leftarrow)$ Assume that $R / I$ is an AG-integral domain with $a b \in I$ for all $a, b \in R$. Then $I+a b=I$ so $(I+a)(I+b)=I$. Since $R / I$ is an AG-integral domain we have $I+a=I$ or $I+b=I$. Then $a \in I$ or $b \in I$. Thus $P$ is a c-prime ideal of $R$.

The next following we define of weakly prime ideal.

Definition 2.8. A proper ideal $I$ of an AG-ring $R$ to be weakly prime if $0 \neq A B \subseteq I$ implies either $A \subseteq I$ or $B \subseteq I$ for any ideals $A, B$ of $R$.

Clearly every prime ideal is weakly prime and $\{0\}$ is always weakly prime ideal of $R$. The following theorem we will study properties

Theorem 2.9. If $I$ is weakly prime but not prime, then $I^{2}=0$.

Proof. Since $I$ is weakly prime (but not prime), there exist ideals $A \nsubseteq I$ and $B \nsubseteq P$ but $0=A B \subseteq I$. Since $I \subseteq A+I$ and $I \subseteq B+I$. But if $I^{2} \neq 0$,. By distributive laws "." over "+" of AG-ring we have

$$
\begin{aligned}
0 \neq I^{2}=I I & \subseteq(A+I)(B+I) \\
& =[(A+I) B]+[(A+I) P] \\
& =A B+I B+A I+I I \\
& \subseteq I
\end{aligned}
$$

which implies $(A+I) \subseteq I$ and $(B+I) \subseteq I$, since $I$ is a weakly prime; that is $A \subseteq I$ or $B \subseteq I$, a contradiction. Hence, $I^{2}=0$.

If $R^{2}=0$, then it is evident that every ideal of $R$ is weakly prime. In particular, if an ideal $I$ of an AG-ring $R$ is weakly prime but not a prime ideal, then every ideal of $I$ as an AG-ring is weakly prime by Theorem 2.9.

Theorem 2.10. Every ideal of an AG-ring $R$ is weakly prime if and only if for any ideals $A$ and $B$ of $R, A B=A, A B=B$, or $A B=0$.

Proof. Suppose that every ideal of $R$ is weakly prime. Let $A, B$ be ideals of $R$. Then $A B$ is a left ideals of $R$, if $A B \neq R$, then by hypothesis, $A B$ is weakly prime. We are consider two situation, that is $A B=0$. or $A B \neq 0$. If $0 \neq A B \subseteq A B$, then by Definition 2.8, we have $A \subseteq A B$ or $B \subseteq A B$. Since $A$ and $B$ are ideals of $R$, we have $A B \subseteq A$ and $A B \subseteq B$. Therefore $A=A B$ or $B=A B$. If $A B=R$, then we have $A=B=R$ whence $R^{2}=R$. 
Conversely, let $K$ be any proper ideal of $R$ and suppose that $0 \neq A B \subseteq K$ for ideals $A$ and $B$ of $R$. Then we have either $A=A B \subseteq K$ or $B=A B \subseteq$ $K$.

Corollary 2.11. Let $R$ be an-AG-ring and every ideal of $R$ is weakly prime. Then for any ideal $I$ of $R$, either $I^{2}=I$ or $I^{2}=0$.

The following theorem we will study relation of c-prime, 3-prime, weakly prime ideals.

Theorem 2.12. Every c-prime ideal is a weakly prime ideal

Proof. Suppose that $I$ is a c-prime ideal of AG-ring $R$. By Lemma 2.3 we have $I$ is a prime ideal. Since every prime ideal is weakly prime ideal we have $I$ is a weakly prime ideal of $R$.

Theorem 2.13. Every 3-prime ideal is a weakly prime ideal

Proof. Suppose that $I$ is a 3 -prime ideal of AG-ring $R$. By Lemma 2.4 we have $I$ is a prime ideal. Since every prime ideal is weakly prime ideal we have $I$ is a weakly prime ideal of $R$.

\section{Acknowledgments}

The author is very grateful to the anonymous referee for stimulating comments and improving presentation of the paper.

\section{References}

[1] K.B. Srinivas, C-Prime Fuzzy Ideals of $R^{n}[x]$, Int. J. Contemp. Math. Sciences, 8 (2013), 133-137.

[2] M.A. Kazim, and MD. Naseeruddin, On almost semigroup, Portugaliae Mathematica, 36(1977), 41-47.

[3] M. Khan, Faisal, and V. Amjid, On some classes of Abel-Grassmann's groupoids, arXiv:1010.5965v2 [math.GR], 2(2010), 1-6.

[4] Q. Mushtaq and M. Khan, M-System in LA-semigroups, Southeast Asian Bulletin of Mathematics, 33(2009), 321-327. 
[5] M. Sarwar (Kamran), Conditions for LA-semigroup to resemble associative structures, PhD thesis, Quaid-i-Azam University, 1993.

[6] T. Shah and I. Rehman, On LA-rings of finitely nonzero function, Int. J. Contemp. Math. Sciences, 5(2010), 209-222.

[7] Y. Hirano, E. Poon, and H. Tsutsui, On rings in which every ideal is weakly prime, Bull. Korean Math. Soc., 47(5)(2010), pp. 1077-1087. 